Archives

$16 \mid 1996$

Pour une histoire comparée du vœu

\title{
L'effraction de s'ordonner. Note sur la conclusion du vœu monastique selon la Règle du Maître
}

\section{Pierre-Antoine Fabre}

\section{Q OpenEdition}

Journals

Édition électronique

URL : http://journals.openedition.org/ccrh/2638

DOI : $10.4000 /$ ccrh.2638

ISSN : $1760-7906$

Éditeur

Centre de recherches historiques - EHESS

Édition imprimée

Date de publication : 16 avril 1996

ISSN : 0990-9141

Référence électronique

Pierre-Antoine Fabre, "L'effraction de s'ordonner. Note sur la conclusion du vœu monastique selon la Règle du Maître ", Les Cahiers du Centre de Recherches Historiques [En ligne], 16| 1996, mis en ligne le

27 février 2009, consulté le 19 avril 2019. URL : http://journals.openedition.org/ccrh/2638 ; DOI :

$10.4000 /$ ccrh. 2638

Ce document a été généré automatiquement le 19 avril 2019

Article L.111-1 du Code de la propriété intellectuelle. 


\title{
L'effraction de s'ordonner. Note sur la conclusion du vœu monastique selon la Règle du Maître
}

\author{
Pierre-Antoine Fabre
}

1 L'objectif de cette brève contribution, jalon d'un travail en cours, est de préciser les rapports de l'énoncé d'une règle concernant l'engagement du vœu religieux avec les modalités de l'énonciation de cet engagement, telles que ce même énoncé les désigne, à partir d'un détail de la Règle du Maître, l'un des textes constitutifs de la tradition du monachisme occidental.

2 Je résume deux préalables de cette enquête ${ }^{1}$ :

\section{Du point de vue d'une théorie générale du vœu}

3 Reprenons rapidement les pages décisives consacrées au vœu par Émile Benveniste dans son Vocabulaire des institutions indo-européennes ${ }^{2}$. Le votum se définit tantôt comme le vœu d'obtenir tel ou tel bénéfice, tantôt comme celui d'accomplir tel ou tel acte ; il est « tantôt le souhait que le priant demande à la divinité d'exaucer, tantôt ce qu'il promet à la divinité d'accomplir ». Tantôt il appelle la grâce d'obtenir ; tantôt il rend grâce d'avoir obtenu. Mais ces deux axes peuvent se nouer : je forme un vœu à l'obtention duquel je répondrai par l'accomplissement d'un autre vœu. Le vœu religieux serre d'un nouveau tour cette convergence, puisque l'accomplissement de la profession s'y définit comme la perpétuation d'un vœu. Autrement dit: je fais le vœu de m'acquitter d'un vœu dont l'objet ne sera autre que l'engagement d'un vœu. Qu'est-ce que cela veut dire? Que le vœu religieux substitue un engagement à un acquittement - certes; mais aussi qu'il fait de cet engagement le substitut d'un acquittement. Or, le vœu religieux, en tant qu'il n'est jamais que le moyen, l'instrument, l'occasion de la réalisation d'un vie chrétienne parfaite (Thomas d'Aquin, Somme théologique, II, II ae, Qu. 186, art. 2 : « L'état religieux est le moyen de l'accès à la perfection»), ne peut pas ne pas faire revenir l'acquittement 
(j'accomplis le vœu d'être religieux et ce vœu m'acquitte de ma dette envers Dieu) dans la doublure de l'engagement (j'accomplis le vœu d'être religieux et ce vœu m'engage envers des hommes). On le comprendra donc: le temps de latence de l'accomplissement, ce temps dans lequel le futur religieux fait vœu d'obtenir et d'accomplir (et donc d'obtenir sans que cette obtention projette encore l'ombre d'un acquittement, sans que le vœu contracté sécrète le processus de sa propre dissolution), ce temps décide de la force du lien. L'histoire du vœu religieux ne cesse de le montrer : c'est dans la dilation, dans le retardement de l'accomplissement du vœu, dans l'espacement de temps du vœu du vœu, que les communautés prennent corps. L'énonciation du vœu, en tant qu'elle demeure suspendue dans l'effectuation de son acte, fait venir dans sa durée propre, sa durée spécifique d'acte de langage, ce que nous découvrons comme la temporalité fondamentale du vœu religieux, identification et différemment du vœu d'obtenir et du vœu d'accomplir.

\section{Du point de vue d'une histoire du vœu religieux}

4 Ce n'est pas seulement de chaque ordre ou de chaque institution particulière, positivement différenciés de tous les autres par tel ou tel trait spécifique, que la dilation de l'intégration, ce que l'on appelle le temps de probation, définit le contour et garantit la stabilité ; il en va, bien au-delà, de l'inscription de tel ou tel de ces ordres particuliers dans l'histoire générale des institutions religieuses en tant qu'elles ne désignent jamais une fin propre, mais qu'elles sont l'instrument d'une fin commune, qu'elles ne cessent de «fonder» et de «refonder» une même institution, que seuls les écarts-les malentendus - de la transmission feuillettent en plusieurs formations distinctes.

5 Le paradoxe étant que la formulation du vœu ou, plus généralement, de la volonté de prononcer ses vœux, bien qu'elle revête une importance fondamentale dans la détermination de l'institution, se trouve nécessairement reléguée à la marge du discours par lequel elle s'autorise; et ceci non seulement parce que les rituels d'insertion découpent par définition un espace périphérique, entre dedans et dehors, une zone de frontière, mais encore parce que l'oralisation essentielle de l'énoncé de la règle dans le procès de son énonciation soustrait cet énoncé, comme vecteur dynamique de l'incorporation - de l'incorporation du postulant dans la communauté, de l'incorporation des énoncés de la communauté par le postulant -, à l'établissement « définitif » du texte constitutionnel.

6 L'examen des chapitres consacrés à l'entrée en probation dans les trois premières grandes règles du monachisme chrétien - les Institutions cénobitiques attribuées à Jean Cassien, la Règle du Maître et la Règle de saint Benoit - nous montre la faille dans laquelle s'engouffre la formulation du vœu du postulant; trois règles échelonnées $d u v^{\mathrm{e}} \mathrm{au} \mathrm{VI}^{\mathrm{e}}$ siècle et entre lesquelles la construction du mythe d'une transmission orale malentendue produit un certain nombre d'effets déterminants, par la répercussion transformée d'énoncés dont la variation contribue à fonder l'instance d'une source orale de l'inscription (de la "dictée ») de la règle, en même temps qu'elle s'efforce de la résorber dans le marquage textuel des rituels d'énonciation orale. Je ne développe pas, ici, plus avant ce schéma général.

7 Rappelons le cadre du détail de la Règle du Maître sur lequel je ferai ensuite porter le travail, pour montrer comment, au sein d'un même texte, l'énoncé désigne le rituel du vœu comme lieu d'énonciation. Comme dans les Institutions cénobitiques de Cassien et dans 
la Règle de saint Benoît, la juridiction de l'insertion dans la communauté intervient au terme de la règle : "Comment un frère, soit déjà convers, soit encore laïc, doit entrer au monastère, s'y offrir et y être reçu » (chap. 87) ${ }^{3}$. Cette situation place l'entrée dans l'ordre à un point de bascule entre, d'une part, la résorption de la prescription - que doit-il en être de l'effectivité de l'ordre par rapport à sa règle? - dans une description de la vie de l'ordre comme corps constitué, description au sein de laquelle l'insertion dans l'ordre d'un nouveau membre relève naturellement de ses règles de reproduction, et n'intervient donc, tout aussi naturellement, qu'au terme de la présentation de cet ordre, et, d'autre part, la marque d'une limite de l'énoncé de la règle, au-delà de laquelle, pour celui qui postule à son insertion, la règle devra être répétée, réénoncée. L'article de l'insertion est donc à la jointure de la règle instituée - énoncée dans le texte qui la fixe - et de l'énonciation de cette règle. Aussi bien le chapitre 87 s'amorce-t-il de la manière suivante : "Quand [le postulant] aura dit qu'il est capable d'obéir en tout, alors on lui lira la présente règle du monastère [...] Alors l'abbé poursuivra en disant : " Qu'adviendra-t-il de tes biens, dont tu disposes à ton gré [...] Mieux vaut, comme dit l'écriture, que 'ton trésor soit aussi là où est ton cœur' $»^{4}$. Trois guillemets qui referment une citation (la nôtre) dans laquelle l'abbé - celui qui reçoit - est intervenu dans la règle même dont il a, aussi, donné la lecture : l'inscription expresse de l'énonciateur dans le texte d'une règle qu'il vient d'énoncer trouve sa marque spécifique dans l'acte d'une énonciation insérée à ce second niveau: la citation d'un passage de l'écriture (à deux reprises dans ce même paragraphe). Ce relais scripturaire porte en vérité le passage de l'énonciation référée de la règle (" on lui lira la présente règle ») à l'énoncé, cité, de paroles de l'abbé : il autorise l'énonciation de la règle, par l'abbé d'abord, puis, comme nous le verrons, par le postulant, en adossant cette règle à l'écriture dont tout énoncé se doit de procéder ; il distend donc la règle entre son énoncé - renvoyé à l'écriture - et son énonciation, désignée par là-même comme une modalité de ré-énonciation de l'écriture.

Cette première opération réalisée, la Règle du Maître pourra confirmer l'ouverture d'un lieu d'énonciation radicalement soustrait à l'énoncé de la règle : « Une fois achevé le délai de deux mois donné pour délibérer, si, la discipline leur plaisant, ils choisissent plutôt la stabilité, et qu'après une nouvelle promesse de s'attacher à la règle qu'on leur a lue ( repromissa lectae regulae firmitate), il leur plaît de garder la persévérance [...] $»^{5}$. Une nouvelle promesse, dont nous ne trouvons nulle trace dans l'épisode précédent, qui est pourtant bien celui lors duquel la règle a été lue au(x) postulant(s). La promesse affleure dans l'énoncé de la règle comme promesse répétée, après une première promesse, tenue dans le secret d'une énonciation en marge, et qui, elle, ne valait que comme promesse d'être répétée, promesse de promettre. Nous touchons ici du doigt le processus, dont je rappelais plus haut les déterminations globales, d'une dilation du temps de l'énonciation de la règle, dilation du temps de l'énonciation dans l'acheminement de l'énoncé de la règle, la promesse s'effectuant, dans ce deuxième temps, comme un énoncé du texte de la règle, et, tout aussi bien, comme la confirmation de la promesse de s'y soumettre.

Dans l'étape qui suit, un dialogue direct (directement décrit, mais au mode futur, par le texte de la règle) confronte le postulant à l'abbé. Ce dialogue se clôt sur deux longs discours, le premier de l'abbé, le second du postulant. Or ces deux discours s'achèvent sur ceci (par quoi je referme le cadre de ce dialogue, sur le détail duquel je vais revenir) : " Après ce discours (Post haec dicta), le frère nouveau dira lui-même ce répons [...] $»^{6}$. Or, le discours du postulant peut intervenir ou non selon que celui-ci entre ou non « avec ses biens ». Le « haec» de " post haec dicta» renvoie donc de fait (comme le remarque bien le 
commentaire critique d'Adalbert de Vogüé, mais sans, me semble-t-il, en prendre toute la mesure) aussi bien au discours de l'abbé qu'à celui du postulant. En ce point, deux énonciateurs partagent l'énoncé de la règle: cet énoncé est transmis, à travers la transmission d'une énonciation dont l'appartenance reste ici secrète, en réserve de l'énoncé, dans un lieu commun dont cet énoncé ne discerne pas les propositions qui s'y trouvent produites.

Or venons maintenant, au cœur de ce passage par lequel le postulant investit le lieu de l'énonciation de la règle à laquelle l'énoncé de cette règle le soumet, à la confrontation du maître de la communauté et de son futur membre. Ce dialogue marque le seuil décisif de l'entrée dans l'ordre, au-delà duquel le discours de l'abbé conclura l'admission du postulant. Il va donc ici du terme d'un parcours dont le premier temps, comme je l'ai rappelé, était la lecture de la règle de la communauté au postulant et la première promesse (secrète) qu'il avait faite d'obéir à cette règle. Il s'agit donc bien de l'accomplissement du vœu d'insertion.

Or, nous allons le voir, c'est par effraction que le postulant accomplit ce vœu, dont, mieux encore, la formule demeure interdite. La scène a lieu au «lendemain de l'énoncé » de la « nouvelle promesse »: «Après qu'on aura fini de dire les prières de prime, quand l'abbé sortira avec la communauté sur le seuil de l'oratoire [...] le disciple le saisira par le vêtement et le retiendra humblement avec la main, en lui présentant la demande suivante : «J'ai quelque chose à suggérer, d'abord à Dieu et à ce saint oratoire, puis à toi et à la communauté. » L'abbé répondra : « Fais-nous connaître ce que c'est. » Le postulant (petitor) poursuivra : «Je veux servir Dieu par la discipline de la règle qui m'a été lue, dans ton monastère. $" »^{7}$.

Remarquons ici, avant d'aller plus loin, que le texte latin donne : "Volo Deo servire per disciplinam regulae mihi lectae in monasterio tuo." D'où deux leçons possibles, entre lesquelles la traduction précédemment citée, du reste, se garde de choisir ; soit : « je veux servir Dieu, dans ton monastère, par la règle qui m'a été lue »; soit : «Je veux servir mon Dieu par la règle qui m'a été lue dans ton monastère ». Le lieu de l'application de la règle est aussi le lieu de l'énonciation de la règle, ou, plus précisément : le lieu de l'application de la règle est d'abord le lieu de son énonciation. La règle n'est pas celle du monastère, mais celle qui, entendue dans ce monastère, sera obéie dans ce monastère. Par où nous voyons, très clairement marquée, la part de l'énonciation de la règle dans sa spécification comme règle de ce monastère-ci, et la place du moment de cette énonciation dans la singularisation de cette institution particulière.

Poursuivons et voyons comme le développement de l'échange va consacrer le monastère comme lieu d'énonciation : « [...] « Je veux servir Dieu (Volo Deo servire) par la discipline de la règle qui m'a été lue dans ton monastère. » L'abbé répondra : «Tu le veux (Et hoc placet tibi) ?» Le futur disciple (futurus discipulus) poursuivra : «C'est Dieu qui le veut d'abord, et moi ensuite (Hoc primo Deo, sic et mihi). » Alors l'abbé dira : « Vois frère, ce n'est pas à moi que tu promets (mihi nihil promittis), mais à Dieu et à cet oratoire et à ce saint autel » $»^{8}$.

Que constatons-nous? Cet étrange dialogue, ouvert dans une brèche violemment forcée par le postulant saisissant l'abbé par son vêtement (comme si l'échange n'aurait pas dû avoir lieu, comme si le postulant avait dû forcer l'énoncé de la règle, le cours ordinaire de la cérémonie pour se faire une place), se poursuit de telle sorte qu'à aucun moment ce dialogue - que j'ai ainsi improprement nommé - ne s'instaure. Une succession de malentendus (que la traduction d'Adalbert de Vogüé s'efforce d'aplanir) en marque en effet la progression. $\mathrm{Au}$ « volo» du postulant, l'abbé répond par un «placet » : le «volo» 
«donne lieu au sujet parlant $»^{9}$, sans que cette position trouve le répondant d'une proposition ; mais, inversement, la proposition par laquelle l'abbé n'entend pas ce «volo » reste elle-même sans réponse chez le postulant, sans que celui-ci revienne pour autant à l'énoncé de sa proposition initiale: des mots de l'abbé, il ne retient que le «hoc» qui renvoie autant à ce que l'abbé suivait d'un " placet » qu'à ce que lui-même précédait d'un « volo » : « hoc primo Deo, sic et mihi ». Ce que, à son tour, l'abbé ne retient qu'en le répétant - comme le " hoc » précédent - par un nouvel écho, un nouveau partage du signifiant - « mihi »- dont l'effet de malentendu apparaît comme la conséquence même : "mihi nihil promittis».

Mais, c'est en même temps au terme de ces transferts et de cette écoute malentendue des signifiants par deux sujets dont les propositions n'articulent pas la différence des positions, que se trouve désignée leur position commune, devant « cet oratoire et ce saint autel ». L'abbé se démet de la réception d'une proposition qu'il entend mal (le postulant ne lui a rien promis), mais, se démettant, découvre le lieu de leur position désormais commune (le postulant n'étant déjà plus le «petitor » du début du dialogue mais un " futurus discipulus ", non plus le disciple de l'abbé, comme dans le début de la séquence, mais le futur disciple de la règle), et découvre ce lieu comme le lieu commun d'une énonciation livrée à l'échange d'un corps d'énoncés réglés par un texte qui en perpétue les opacités, et auquel s'ordonne cependant un vœu - un « volo » - reçu comme " placet »; comme "placet tibi»: toi, qui ne faisais qu'un avec ce «volo», par lequel tu étais le «vouloir » d'un «dire », je te réinstitue dans l'ordre de la langue : il te plaît à toi, sujet d'un verbe, de servir Dieu. Je t'ai mal entendu, mais je t'ai compris, et je t'ai admis, ici, en ce lieu où tu as voulu.

\section{NOTES}

1. Je reprends, dans les deux paragraphes qui suivent, les acquis d'une première approche : P.-A. Fabre, « Prononcer ses vœux », L'Inactuel, Paris, automne 1995, p. 121-130.

2. Paris, Minuit, 1969 , tome 2, p. 232-243.

3. Règle du Maître, Sources chrétiennes, $\mathrm{n}^{\circ}$ 106, p. 354-355.

4. Ibid., p. 356-357.

5. Ibid., p. 370-372.

6. Ibid., p. 376-377.

7. Ibid., p. 372-373

8. Id.

9. Michel de Certeau, La Fable mystique, Paris, 1982, p. 240. 


\section{AUTEUR}

\section{PIERRE-ANTOINE FABRE}

CRH, Paris 